\title{
Psychosozialer Notfall Substanzinduzierte Störungen durch illegale Drogen - Teil 2
}

Michael Kinn • Rüdiger Holzbach • Frank-Gerald Bernhard Pajonk

\begin{abstract}
Drogenintoxikationen sind ein relevantes Thema für Notärzte: Zum einen, weil sie oft schwer zu beherrschen sind - zum anderen, weil ihr Auftreten alles andere als rückläufig ist. Die Zahl der Drogentoten stieg 2007 um 8\% gegenüber dem Vorjahr. Der erste Teil dieser Serie berichtete über die häufigen Drogen Cannabis, Kokain und Heroin (Anästhesiol Intensivmed Notfallmed Schmerzther 2008; 43: 746-753). Dieser zweite Teil behandelt nun Notfälle im Zusammenhang mit dem Konsum von Designerdrogen, Medikamenten und Inhalantien.
\end{abstract}

\section{Stimulanzien: Amphetamine und Ecstasy}

Konsumvarianten Amphetamine und Metamphetamine werden oral, nasal, intravenös und inhalativ konsumiert. Sie wirken

- als indirekte Katecholamin-Agonisten durch Freisetzung von Dopamin und Noradrenalin aus präsynaptischen Neuronen und

- in hohen Dosen durch die Hemmung der Monoaminooxidase [1].

Die häufigste Handelsform ist ein weißes Pulver, das geschnupft wird ( $\bullet$ Abb. 1, 2).

Ecstasy Zu der Wirkstoffgruppe, die unter dem Namen Ecstasy bekannt ist, gehören die Derivate der $\beta$-Phenethylamine

- MDMA (3,4-Methylenedioxy-N-methamphetamin),

- MDA (3,4-Methylenedioxy-N-amphetamin),

- MDE (3,4-Methylenedioxy-N-ethamphetamin) und

- MBDB (N-Methyl-1-1,3-benzodioxol-5-yl-2butanamin) [2].

Sie bewirken eine verstärkte Ausschüttung und Wiederaufnahme-Hemmung von Serotonin und Dopamin im synaptischen Spalt. Bei oraler Einnahme tritt die Wirkung nach ca. $30 \mathrm{~min}$ auf und hält 3-6h an [1]. Die Wirkstoffe kommen meist als motivgeprägte Tabletten in Umlauf ( $\bullet$ Abb.3). Sie enthalten neben den Grundstoffen überwiegend noch Zusatzstoffe wie

- Coffein,

- Ephedrin oder
- andere Wirksubstanzen.

- Zusätzlich werden viele weitere Streck- und Bindemittel bei der Herstellung verwendet.

Eine Tablette Ecstasy enthält in der Regel 60-120 mg MDMA oder MDMA-Analogon. Die genaue Zusammensetzung kennt jedoch meist nur der Hersteller.

Rauschwirkung von Ecstasy MDMA führt bei den Konsumenten zu einem angenehmen emotionalen Zustand der Ruhe und Einheit mit der Umgebung, zum gesteigerten Wunsch nach Nähe zu anderen und zu leichten Wahrnehmungsveränderungen.

- Die Aktivierung ist schwächer als bei den Amphetaminen, die sensorischen Veränderungen sind weniger ausgeprägt als bei Halluzinogenen wie LSD oder Meskalin [2].

- Insgesamt wirken die oben genannten Substanzen stimulierend.

Charakteristisch sind Euphorie mit „HighGefühl“, eine subjektiv gesteigerte geistige und körperliche Leistungsfähigkeit, eine erhöhte Vigilanz sowie Unterdrückung von Schlaf, Müdigkeit und Hunger- und Durstgefühl.

Intoxikation Überdosierung und Beigebrauch unterschiedlichster Substanzen können zu Vergiftungen mit zum Teil sehr gemischter Symptomatik führen.

- Besonders häufig kommt es zu Tachykardien, Hypertonie, Mydriasis, Tremor, Palpitationen, Parästhesien, Hyperhidrosis, Hitzewallungen oder erhöhter Kälteempfindlichkeit, zu Übelkeit und Verschwommensehen, psychomotorischer Unruhe, Erbrechen sowie thorakalen Schmerzen.

Exitus nach Konsum Todesfälle infolge Stimulanzien-Einnahme können bedingt sein durch - Rhabdomyolyse mit hohem Fieber, 
- disseminierte intravasale Gerinnungsstörungen und Nierenversagen,

- kardiovaskuläre Zwischenfälle (supraventrikuläre Tachykardien, QT-Intervallverlängerungen mit ventrikulären Tachykardien im Sinne von Torsades de pointes, Myokardinfarkte, Kammerflimmern, Angina pectoris, Asystolie) $[1,3,4]$.

In seltenen Fällen kann es auch zur Ausbildung eines Pneumomediastinums kommen [5].

Weitere somatische Komplikationen können sein: Hypertensive Krisen mit intrazerebralen Mikro- und Makrohämorrhagien, Verwirrtheit, Atemdepression, Krampfanfälle, Dyskinesien, Dystonien, Hyperthermien, Dehydration und Bewusstseinstrübungen bis zum Koma [6].

Psychische Symptome Bei einer Intoxikation mit Stimulanzien kann es zu folgenden Phänomenen kommen:

- Anspannung, Agitiertheit

- psychotische Phänomene (Wahn in Form von Beeinträchtigungs- und Verfolgungswahn, sog. Speed-Paranoia)

- Halluzinationen in Form von optischen und taktilen Mikrohalluzinationen (z. B. „Ameisenlaufen“ oder „Wanzen unter der Haut“)

- Alternieren zwischen ekstatischer Gehobenheit und ängstlich-depressiver Gestimmtheit

Versorgung bei Intoxikation Die notärztliche Therapie ist symptomatisch. Der Auffindeort (Diskothek) und die Aussagen von Patient oder Umfeld können Hinweise auf eine Intoxikation mit Stimulanzien geben.

- Die in einigen Bundesländern bereits verbreiteten Drogenschnelltests können lediglich eine Aussage darüber treffen, ob ein Patient in letzter Zeit Kontakt zu Drogen hatte.

- Eine Bestimmung der akut zugeführten Droge und Menge ist mit ihnen derzeit nicht möglich.

$\mathrm{Zu}$ den Basismaßnahmen im Rahmen einer Stimulanzienintoxikation gehören das kardiolo- gische Monitoring und die Flüssigkeitssubstitution.

- Nitrate scheinen geeignet, hypertensive Krisen zu bekämpfen.

- Kammertachykardien sollten mit Amiodaron therapiert werden.

- Zur Senkung der Körpertemperatur beim hyperthermen Patienten können Kühlpacks in der Leiste (wenn diese toleriert werden) und kalte Infusionslösungen zum Einsatz kommen.

Der ateminsuffiziente oder komatöse Patient muss frühzeitig intubiert werden. Agitierte oder ängstlich-unruhige Patienten sollten mit Benzodiazepinen behandelt werden - wahnhaftes oder psychotisches Erleben sollte hingegen eher mit Antipsychotika therapiert werden.

Wegen der akut gefährdeten Vitalfunktion müssen die Patienten auf eine Überwachungsoder Intensivstation gebracht werden. Nur wenn Erregung, Angst und paranoides Erleben bei stabilem körperlichem Zustand dominieren, ist eine (geschlossene) psychiatrische Station zu empfehlen.

Stimulanzien-Entzug Stimulanzien verursachen nur einen schwachen physischen und psychischen Entzug.

- Meist stehen Konzentrationsstörungen, Zephalgien, Abgeschlagenheit, Schläfrigkeit, depressive Verstimmung und Ängstlichkeit im Vordergrund.

- Gelegentlich können auch körperliche Symptome wie Bradykardien und Hypotonie auftreten [1].

Diese Symptome dauern etwa 2 Wochen an. Bei exzessivem Konsum von Stimulanzien können Denk- und Merkfähigkeitsstörungen auch dauerhaft persistieren. Vereinzelt kommt es bei ehemaligen Konsumenten zu „flash-back“-Phänomenen mit Wahn- und anderen Psychosephänomenen.
Abb. 1, links Amphetamine werden in der Regel in Pulverform gehandelt; zu diesem Zweck werden sie häufig in sog. Apothekerbriefchen abgefüllt.

Abb. 2, Mitte Eine Linie Pep mit dem üblichen Zubehör (Rasierklinge, gerollter 5 Euro Schein). Pep (englisch für „Elan“, „Schwung“) ist eine Szenebezeichnung für Amphetamin (Speed).

Abb. 3, rechts Ecstasy wird meist in Tablettenform verkauft. Die meisten werden mit verharmlosenden Zeichen geprägt. Unten rechts im Bild eine Rarität aus den frühen Anfangszeiten: „Eva“ - die Mutter aller Ecstasy-Tabletten.
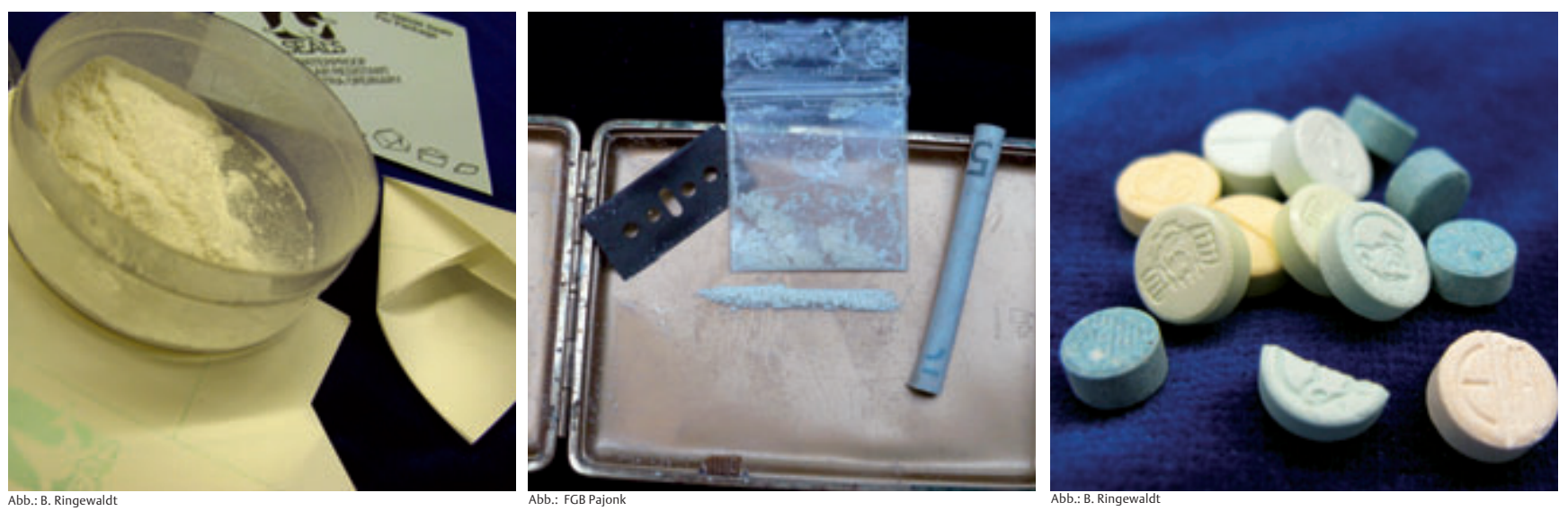
Abb. 4 LSD ist ein halluzinogenes Mutterkornalkaloid. Oben im Bild: Mutterkorn, wie man es zuweilen in Konsumentenwohnungen findet. Darunter: Trips in Papierform. Die perforierten, löschpapierähnlichen Streifen werden in gelöster Substanz getränkt, getrocknet und dann verkauft. Konsumenten legen sich dann den portionierten AbrissStreifen auf die Zunge oder den Augapfel.

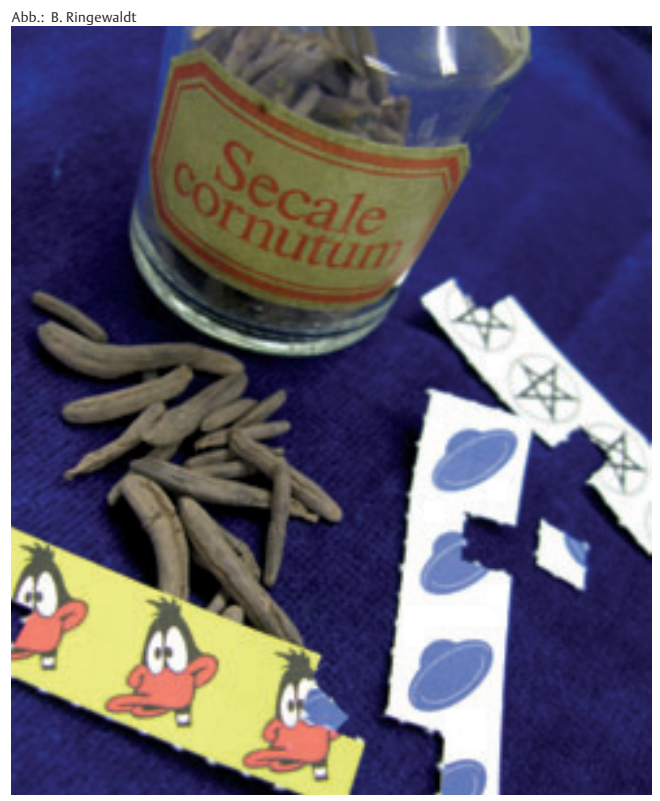

Durch ihre unklare Zusammensetzung geht von synthetischen Drogen ein hohes Gefahrenpotenzial aus. Eine Intoxikation kann von vielen physischen und psychischen Symptomen begleitet sein - mehrgipflige Verläufe sind häufig.

\section{Halluzinogene}

\section{$\nabla$}

LSD und Psilocybin LSD und Psilocybin sind wohl die bekanntesten halluzinogenen Drogen. LSD wird synthetisch hergestellt. Es wurde vom Schweizer Chemiker Albert Hoffmann 1938 bei seinen Forschungen zum Mutterkorn zufällig entdeckt ( Abb. 4).

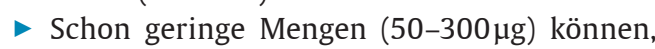
oral eingenommen, zu einem 8-12-stündigen Rausch führen.

- Dabei stehen Halluzinationen oder verändertes Erleben im Vordergrund. LSD wirkt serotonerg über einen agonistischen Effekt am 5 HT2A-Rezeptor.
Psilocybin wird meist in seiner natürlichen Form konsumiert. Es ist in einigen, auch in Deutschland heimischen Pilzen ( $\bullet$ Abb. 5) enthalten und wird oral aufgenommen ( Abb. 6).

- Üblicherweise werden 3-8 Pilze eingenommen, um einen Rausch von 3-6h zu verspüren [1].

Psychoaktive Pilze Zu dieser Gruppe gehört auch der Spitzkegelige Kahlkopf (Psilocybe semilanceata), der ebenfalls in Deutschland auf Viehweiden wächst [7]. Weitere in Deutschland vorkommende psychoaktive Pilze sind u.a. der Blaufärbende Kahlkopf (Psilocybe cyanescens), der Blauende Düngerling (Panaeolus cyanescens), der Fliegenpilz (Amanita muscaria) und der Pantherpilz (Amanita pantherina).

Atypische Halluzinogene Die Inhaltsstoffe von Fliegenpilzen oder Nachtschattengewächsen (z. B. Stechapfel, Engelstrompete) gehören zu den atypischen Halluzinogenen.

- Fliegenpilze enthalten Ibotensäure und das Alkaloid Muscimol, sie wirken auf die GABAergen Mechanismen [6].

- Die verschiedenen Arten von Nachtschattengewächsen (Solanaceae) enthalten TropanAlkaloide, insbesondere Atropin, Hyoscyamin und Scopolamin. Sie rufen anticholinerge Eigenschaften hervor.

Weitere halluzinogene Substanzen sind in - Tab. 1 aufgeführt.

Intoxikation Die oft beschriebene „Bewusstseinserweiterung “ unter halluzinogenen Drogen bezeichnet einen qualitativ veränderten Bewusstseinszustand.

Das Zeiterleben ist deutlich verändert, die Umwelt und die eigene Person werden auf eine traumartige Weise erlebt. Es kommt zu Entgrenzungs- und Verschmelzungserlebnissen, die angenehm bis ekstatisch, aber auch angstvoll erlebt werden können.
Abb. 6, rechts Eine von
vielen Konsummöglichkeiten: das Sandwich mit psychoaktiven Pilzen.

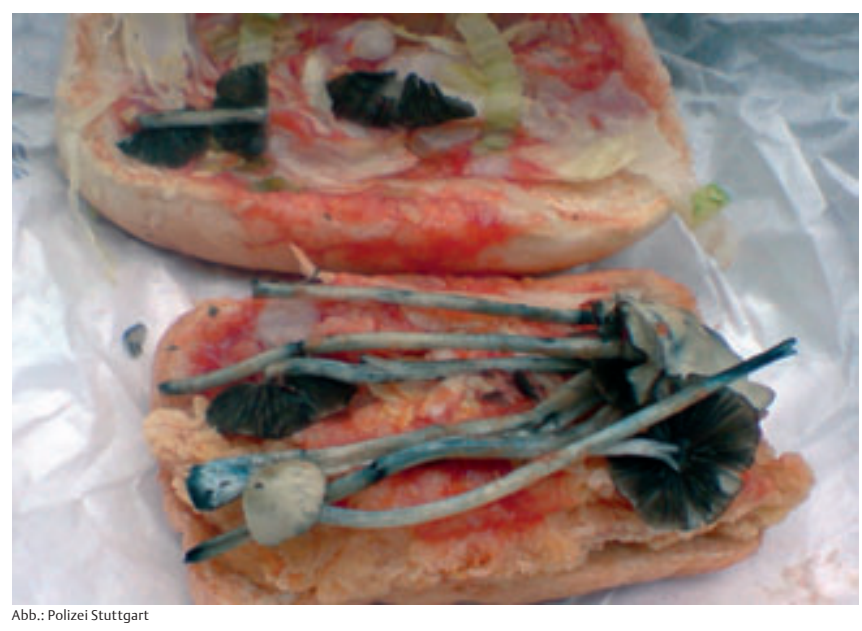

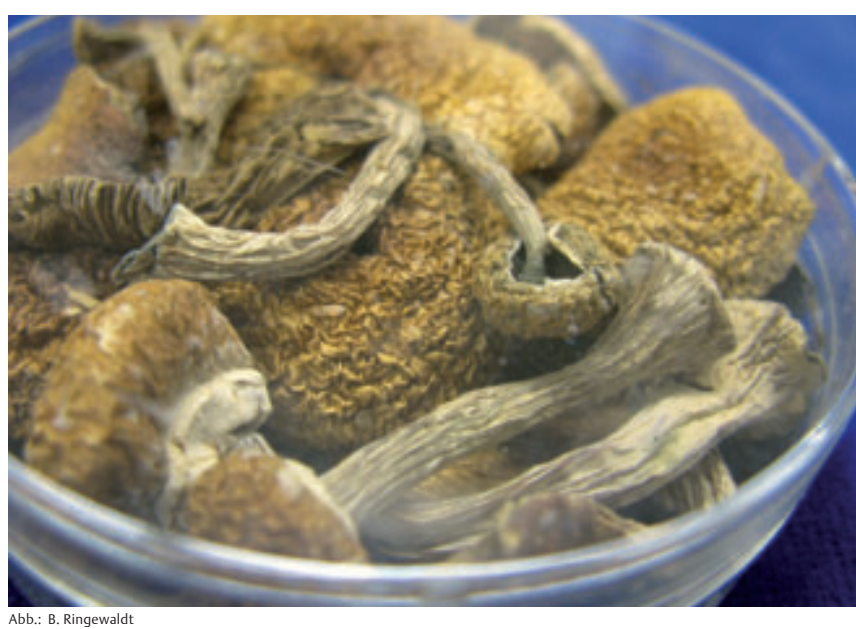


- Die klassischen Halluzinogene rufen selbst bei Überdosierung nur geringe somatische Beschwerden hervor [1].

- Atypische Halluzinogene hingegen können selbst bei üblichen Dosierungen neben den qualitativen Bewusstseinsveränderungen ( $\bullet$ Fallbeispiel) zusätzlich dämpfende und sedierende Effekte bzw. eine Vigilanzminderung hervorrufen [8].

Psychotisches Erleben Meist in höheren Dosierungen können psychotische Rauschverläufe, Ängste und Agitationen (Horror-Trips) auftreten.

- Der regelmäßige Konsum kann drogeninduzierte Störungen hervorrufen, die mehrere Wochen anhalten und möglicherweise in die Spätmanifestation einer schizophrenen Psychose übergehen.

- Noch Wochen bis Monate nach einem Rausch können „Flash-backs“ (Echopsychosen) bzw. persistierende Wahrnehmungsstörungen dazu führen, dass die psychischen Phänomene des Rausches ohne erneute Substanzeinnahme wieder auftreten. Dieser Zustand kann Sekunden bis Minuten anhalten, in ganz seltenen Fällen aber auch wieder anhaltend auftreten $[9,10]$.

Somatische Beschwerden Typisch sind bei der Einnahme klassischer Halluzinogene:

- Mydriasis, Hypertonie, Tachykardie, Hyperthermie sowie Übelkeit und Erbrechen zu Beginn der Rauschwirkung [10].

- Atypische Halluzinogene (z.B. Fliegenpilze, Engelstrompeten) können zu Bewusstseinstrübungen, Orientierungsstörungen und deliranten Symptomen mit lebensgefährlichen vegetativen Entgleisungen, im Extremfall mit Bewusstlosigkeit, Koma und Atemlähmung führen.

Versorgung bei Intoxikation Die oftmals agitierten und verängstigten Patienten müssen beruhigt (,talking down“) und gegebenenfalls mit Benzodiazepinen (z.B. Diazepam 5-20mg i.v.) sediert werden [1]. Psychotische Symptome klingen meist nach kurzer Zeit wieder ab, in besonders schweren Fällen kann die Gabe von Antipsychotika (z.B. Haloperidol 5-10 mg i.v.) erwogen werden. Prüfung und Sicherung der Vitalfunktionen sind obligat.

Halluzinogene verursachen nur eine geringe psychische Abhängigkeit. Physische Entzugserscheinungen sind nicht bekannt. Notfälle können besonders nach Einnahme atypischer Halluzinogene vital bedrohlich werden.

\section{Fallbeispiel}

\section{Der Fall}

Es ist Samstagabend, die Leitstelle nennt als Einsatzgrund eine „verwirrte Person“. Angerufen habe eine 20-Jährige, die berichtet habe, ihr ein Jahr älterer Freund - ein Auszubildender bei einer Bank - sei völlig verwirrt und „durchgeknallt“. Sie habe große Angst um ihn. Der alarmierte Notarzt findet die Freundin vor einem Haus in gutbürgerlicher Wohnlage. Sie berichtet weinend, sie habe ihren Freund zuletzt am Donnerstag gesehen - da sei er nachmittags noch ganz normal - ein eher ruhiger, strebsamer Typ - gewesen. Sie habe besorgt bei ihm vorbeigeschaut, weil er sich nicht gemeldet und auf ihre Anrufe nicht geantwortet habe - das sei sehr untypisch für ihn. Nun wolle sie nicht mehr mit in die Wohnung kommen, weil sie es dort nicht mehr aushalte.

Anamnese, Diagnostik, Therapie Notarzt und Rettungsdienstmitarbeiter finden eine abgedunkelte Wohnung. Das Fußbodenparkett ist zum Teil herausgerissen, auf dem Boden sind Kothaufen und Urin. Die Wände sind teils mit Plastiktüten beklebt, teils kotverschmiert, das Licht funktioniert nicht. Der Patient ist nur mit einer Shorts bekleidet und sitzt grinsend auf dem Boden, seine Füße sind in Müllbeutel eingewickelt. Der Patient kann keine der Fragen (nach Name, Ort, Zeit, was geschehen ist) beantworten, gibt völlig unsinnige und unverständliche Antworten, ist im Gedankengang vollkommen inkohärent. Er lässt sich willig untersuchen. Der körperliche Befund ist weitgehend unauffällig: diskrete Exsikkose, Puls 84/min, RR 120/75 mm Hg, Blutzucker 74 mg/dl, $\mathrm{SO}_{2} 99 \%$. Der Patient ist bereit, mit ins Krankenhaus zu kommen. Im Notarztwagen hält er das Ohr an den Waschbeckenablauf und besteht darauf, ihn zuzukleben. Da er sonst ruhig ist, wird lediglich eine kristalline Infusionslösung über Venenverweilkanüle verabreicht.

Weiterer Verlauf In der Notaufnahme wird zunächst eine organische Diagnostik inkl. CCT durchgeführt; diese ist unauffällig. Das Drogenscreening ist negativ. Nach 3 Tagen in stationär psychiatrischer Behandlung ist der Patient wieder weitgehend geordnet. Er berichtet, dass er einmal im Leben Engelstrompete habe probieren wollen. Er schildert zudem, dass er sich in seiner Wohnung verwanzt gefühlt habe, deshalb das Parkett rausgerissen und die Toilette, die Fenster und die Wände zugeklebt habe, um nicht weiter abgehört und belästigt zu werden. Nach 10 Tagen wird er in psychopathologisch unauffälligem Zustand entlassen.

\begin{tabular}{|c|c|c|}
\hline \multicolumn{3}{|l|}{ Weitere Halluzinogene } \\
\hline $\begin{array}{l}\text { Katecholaminhaltiges } \\
\text { Meskalin }\end{array}$ & Myristicin, Elimicin & Salvinorin \\
\hline $\begin{array}{l}\text { Kommt vor in einem } \\
\text { Kaktus, der in den } \\
\text { westlichen USA und } \\
\text { Mexiko verbreitet ist. } \\
\text { Das Kaktusextrakt } \\
\text { wirkt bei oraler } \\
\text { Einnahme nach } \\
30-90 \text { min, der Rausch } \\
\text { hält bis zu } 10 \mathrm{~h} \text { an. } \\
\text { Er führt zu Mydriasis, } \\
\text { Blutdruckanstieg, er- } \\
\text { höhter Körpertempe- } \\
\text { ratur und verstärkter } \\
\text { Exzitation. }\end{array}$ & $\begin{array}{l}\text { Beide Substanzen } \\
\text { werden aus der } \\
\text { Muskatnuss gewonnen } \\
\text { und wirken psyche- } \\
\text { delisch. } \\
\text { Nebenwirkungen wie } \\
\text { Muskelzittern, Übelkeit } \\
\text { und Erbrechen sind } \\
\text { häufig. }\end{array}$ & $\begin{array}{l}\text { ein hochpotentes } \\
\text { Halluzinogen, das } \\
\text { enthalten ist in den } \\
\text { Blättern des Azteken- } \\
\text { salbei } \\
\text { (Salvia divinorum). } \\
\text { bislang nicht straf- } \\
\text { gesetzlich reglemen- } \\
\text { tiert. }\end{array}$ \\
\hline
\end{tabular}


Abb. 7 Gamma-Butyrolacton (GBL) wird im Körper zu GHB umgewandelt. Es findet in der chemischen Industrie häufig Verwendung und unterliegt keinen strafgesetzlichen Reglementierungen. Die Herstellung von GHB aus GBL und einer weiteren Chemikalie ist selbst Laien möglich.

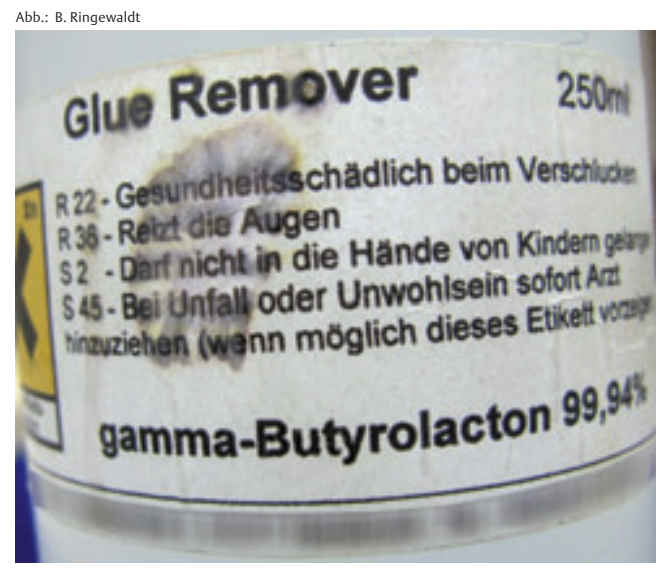

\section{Medikamenten-Missbrauch}

Unter den zugelassenen Medikamenten finden sich einige, die häufig und gern missbräuchlich eingenommen werden. Auf Benzodiazepine und Barbiturate wird dabei in diesem Beitrag nicht eingegangen.

\section{Gamma-Hydroxy-Butansäure (GHB) | Liquid-Ecstasy \\ $\nabla$}

Party- und Bodybuilderdroge GHB (๑ Abb. 7) schöpft sein Missbrauchspotenzial aus der stimulierenden Wirkung. Die Einnahme in niedrigen Dosen führt zu

- Euphorie, Antriebssteigerung, Entspannung und Glücksgefühl, einer vermehrten Kontaktfreudigkeit und Distanzlosigkeit sowie einem erhöhten sexuellen Interesse.

Medizinisch wird GHB bei der Narkolepsie eingesetzt, seit 2005 fällt es unter die BTM-Verordnung.

Wirkungsweise Nach Einnahme werden besonders hohe Konzentrationen in den Basalganglien und im Hypothalamus erreicht. GHB wirkt an den $\mathrm{GABA}_{\mathrm{A}^{-}}$und $\mathrm{GABA}_{\mathrm{B}}$-Rezeptoren [11]. Außer in der Partyszene, wo man es wegen seiner stimulierenden Wirkung einnimmt, wird es auch in der Bodybuilderszene zur Fettreduktion missbräuchlich eingesetzt. In höheren Dosierungen wirkt GHB narkotisierend. Es wird in $12-24 \mathrm{~h}$ vollständig metabolisiert und ausgeschieden, was den Nachweis einer GHB-Intoxikation erschwert.

GHB steht als „K.O.-Tropfen“ oder „Date-RapeDrug“ immer wieder in Zusammenhang mit Sexualdelikten.

K.O.-Tropfen „GHB“ Bei zweifelhaften Auffindesituationen (entkleidete Patientin, Anzeichen für sexuelle Handlungen, fremdanamnestische Berichte über plötzliches stark verändertes Ver- halten, dass nicht durch andere Ursachen wie z. B. Alkoholkonsum erklärbar ist) sollte stets die Polizei hinzugezogen werden.

- Wichtig ist das frühzeitige Asservieren von Körperflüssigkeiten, denn GHB wird schnell abgebaut und ist dann nicht mehr nachweisbar.

- Die Opfer leiden oft unter einer retrograden Amnesie und können so meist keine Angaben zum Verbrechen oder zum Tathergang machen.

Symptome der Intoxikation Übelkeit und Erbrechen, unklare Bewusstseinsstörungen (ähnlich dem Alkoholrausch) und Koma können bei einer Intoxikation mit GHB auftreten.

Notärztliche Versorgung Die Therapie ist symptomatisch orientiert.

- Bei agitierten Patienten können Benzodiazepine (z.B. Diazepam 5-10 mg i.v.) verabreicht werden.

- Antiemetika (z.B. Metoclopramid $10 \mathrm{mg}$ i.v.) können bei anhaltendem Erbrechen zum Einsatz kommen.

- Bei schweren, gesicherten GHB-Intoxikationen kann eine Antagonisierung mit Physiostigmin (1-2 mg i.v.) versucht werden [11].

Die Symptome einer GHB-Intoxikation imitieren oftmals eine Alkoholintoxikation. Die Differenzialdiagnose ist schwierig. Diskrepanzen zwischen fremdanamnestischer Trinkmenge und Befund sollten hellhörig machen.

\section{Ketamin}

v

Konsum steigt Ketamin erfreut sich in der Drogenszene einer wachsenden Beliebtheit. Es wird in Pulverform geschnupft oder als salzwässrige Lösung i.m. injiziert. Die Rauschdosis liegt dabei deutlich unter der narkotischen Dosis. Eine i.v.-Injektion oder die Überdosierung bergen das Risiko des Atemstillstandes.

- Der eigentliche „Trip“ setzt nach etwa 5 min ein und dauert ca. $30 \mathrm{~min}$.

- Restwirkungen können noch nach $2 \mathrm{~h}$ spürbar sein.

- Ketamin wirkt unter anderem am GlutamatNMDA-Rezeptorkomplex im ZNS.

Intoxikation Die missbräuchliche KetaminEinnahme führt zu

- einem dissoziierten Körpergefühl mit bizarren Wahrnehmungen

- bis hin zur empfundenen Körperzerstörung und Todesvisionen.

Die Konsumenten fallen durch Gang- und Bewegungsstörungen auf.

Problematisch ist der Mischkonsum mit Benzo- 
diazepinen oder Alkohol. Er kann zur Entstehung eines „K-Hole“ („Ketamin-Loch“; SzeneAusdruck für eine Überdosierung) führen, verbunden mit Bewegungsunfähigkeit und $\mathrm{Ab}$ schottung von allen Sinneswahrnehmungen. Dieser Zustand gleicht äußerlich einer tiefen Bewusstlosigkeit.

Notärztliche Versorgung Im Vordergrund der Versorgung stehen die Sicherung der Vitalfunktionen und das beruhigende Eingehen auf den Patienten.

Ketamin-Intoxikationen können besonders durch die Kombination mit anderen Suchtmitteln sehr unangenehme und zudem vital bedrohliche Zustandsbilder hervorrufen.

\section{Phenylcyclidinpiperidin (PCP)}

„Angel Dust“ PCP wurde um 1963 als dissoziatives Anästhetikum entwickelt, aber bereits nach 2 Jahren wieder vom Markt genommen. Es ist bekannt als „Angel Dust“, auf den Markt kommt es als

- weißes Pulver, Flüssigkeit oder Gas, und es wird

- geraucht, geschnupft, oral eingenommen oder inhaliert.

Rausch-Erleben Der Rausch wird als euphorisierend oder unruhig und nervös beschrieben, er hält bis zu $48 \mathrm{~h}$ an. Dabei kommt es zu einem dissoziierten Körpergefühl, und es können panische Ängste auftreten. Bei hohen Dosen dominieren Aggression und die Fehleinschätzung realer Gegebenheiten, was in eigen- oder fremdgefährdenden Situationen münden kann.

Intoxikation Somatische Symptome sind Tachykardie, Hypertonie, starrer Blick mit weit aufgerissenen Augen, Tränen- und Speichelfluss und Muskelversteifungen.

- Eine Intoxikation mit PCP kann zu Krampfanfällen, Hypotonie und Bewusstlosigkeit bis zum tagelangen Koma und schließlich Tod durch Herzkreislaufversagen führen.

Notärztliche Versorgung Der Patient wird symptomatisch behandelt und sollte von Umwelteinflüssen abgeschirmt werden. Ein „talking down“ kann zur Steigerung von Aggression, Unruhe und Angst führen - daher empfiehlt sich bei Unruhe und Erregung der frühzeitige Einsatz von Benzodiazepinen.

\section{Schnüffelstoffe}

Narkosegase (Lachgas, Halothan)

organische Lösungsmittel

Gase in Haushalts- und Gewerbeprodukten

Aerosole

aliphatische Nitrite (Amylnitrit, Butylnitrit)

Klebstoffe

Treibmittel

Arzneimittel

Reinigungsmittel und Lösungsmittel

Kraftstoffe

Tab. 2

Cave Der Notarzt sollte sich einem Patienten mit PCP-Intoxikation behutsam nähern! Bei deutlich agitierten oder aggressiven Patienten hat der Eigenschutz Vorrang.

\section{Inhalantien}

$\nabla$

Sammelbegriff Als „Schnüffelstoffe“ werden zahlreiche Substanzen ( $\odot$ Tab. 2) bezeichnet, deren Inhalation einen wenige Minuten anhaltenden Rauschzustand hervorrufen kann. Durch Wiederholung können diese Rauschzustände verlängert werden, gleichzeitig steigt aber das Risiko einer Hypoxie mit nachfolgender Bewusstlosigkeit. Weitere Komplikationen sind:

- Übelkeit und Erbrechen mit nachfolgender Aspiration

- Asthmaanfälle

- Lungenödem

- direkte Lähmung des Atemzentrums

Schnüffelstoffe werden häufig von Kindern und Jugendlichen aus sozial schwachen Schichten konsumiert.

Inhalantien bergen viele Gefahren. Besonders die Aspiration mit anschließender Hypoxie fordert bei dem meist heimlichen Konsum durch Kinder und Jugendliche Todesopfer.

Intoxikation Patienten mit einer InhalantienIntoxikation sind häufig antriebsgemindert, psychomotorisch verlangsamt, apathisch und/ oder teilweise aggressiv gespannt. Die Urteilsund Kritikfähigkeit ist herabgesetzt, es sind mnestische und kognitive Defizite feststellbar.

- Neurologisch-internistisch können Hypertonie, Schwindel, Nystagmus, Koordinationsstörungen, eine verwaschene Sprache, Gangunsicherheit, Tremor, Hyporeflexien, Muskelschwäche, verschwommenes Sehen und Doppelbilder vorhanden sein.

- Manchmal sind die Patienten stuporös oder komatös [6]. Bei chronischen Konsumenten können paranoid-halluzinatorische Bilder auftreten. 
Notärztliche Versorgung Auch hier sind die Sicherung der Vitalfunktionen und eine symptomorientierte Therapie indiziert.

- Bei hypertensiven Krisen sollte man einen $\alpha 2$ Agonisten (z.B. Clonidin 0,15-0,3 mg i.v.) geben,

- bei Ängsten und Unruhezuständen kann ein Benzodiazepin (z.B. Diazepam 5-10 mg i.v.) gegeben werden,

- bei psychotischen Symptomen kann man ein Antipsychotikum (z.B. Haloperidol 5-10 mg i.v.) geben.

Bei Ateminsuffizienz und zum Aspirationsschutz sollte eine Intubation frühzeitig erfolgen.

Cave Beim Einsatz von Katecholaminen ist besondere Vorsicht geboten! Einige Substanzen können die Wirkung von Katecholaminen unkontrolliert verstärken!

\section{Mischintoxikationen}

$\nabla$

Kombinationen sind häufig Bei jeder unklaren - aber auch bei vermeintlich klarer - Intoxikation ist an eine Mischintoxikation zu denken. Gerade langjährige Konsumenten greifen mitunter zu festen Abfolgeschemata in der Kombination von Rauschmitteln.

- Beliebt ist ein Abfolgekonsum von Stimulanzien (z.B. Ecstasy, Kokain), denen später sedierende Substanzen (z.B. Alkohol, Benzodiazepine) folgen.

Besonderes Gefahrenpotenzial Unterschiedliche pharmakologische und pharmakokinetische Eigenschaften führen zu schwer einschätzbaren Notfallsituationen. Besonders bei Suizidversuchen sind Mischintoxikationen häufig und gefährlich.

- Bei der notärztlichen Versorgung steht die Sicherung der Vitalfunktionen im Vordergrund.

- Ateminsuffiziente Patienten sollten frühzeitig intubiert werden.

- Die weitere Behandlung erfolgt symptomatisch.

Ein Antidot kann zwar differenzialdiagnostisch hilfreich sein, sollte aber dem absoluten Notfall vorbehalten bleiben, weil dadurch weitere Probleme entstehen können. Sinnvoll ist das Asservieren von Körperflüssigkeiten zur späteren toxikologischen Bestimmung und zur gezielten Weiterbehandlung in der Klinik.

Mischintoxikationen sind häufig und bergen besondere Gefahren. Die Sicherung der Vitalfunktionen hat Priorität. Im Rahmen von Suizidversuchen liegen Mischintoxikationen besonders häufig vor.
Fazit Insbesondere Designerdrogen und missbräuchlich eingenommene Medikamente bergen die Gefahr, unvorhersehbare Rauschverläufe und mitunter lebensbedrohliche Zustandsbilder hervorzurufen. Vorsicht ist auch bei vermeintlich eindeutigen Fällen von Substanzmissbrauch angebracht. Der Mischkonsum ist häufig und sollte stets in Erwägung gezogen werden. Suizidale Handlungen kommen oftmals im Zusammenhang mit Substanzmissbrauch vor. Dem intoxikierten wie dem entzügigen Süchtigen sollte im Notarztdienst stets die volle Aufmerksamkeit des Notarztes zuteil werden, um lebensbedrohliche Situationen zu erkennen und zu beherrschen. $<$

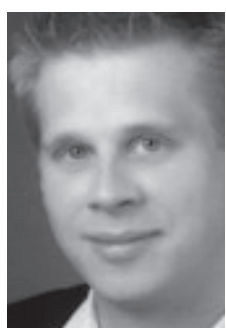

Michael Kinn ist Arzt in Weiterbildung der Abteilung für Anästhesie, Intensivmedizin und Schmerztherapie an der Berufsgenossenschaftlichen Unfallklinik Ludwigshafen. E-Mail: mkinn@bgu-ludwigshafen.de

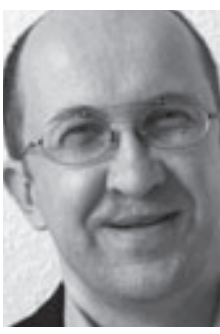

Dr. med. Rüdiger Holzbach leitet die Abteilung Suchtmedizin der Westfälischen Kliniken Warstein und Lippstadt. Sein wissenschaftlicher Schwerpunkt liegt auf dem Thema der Medikamentenabhängigkeit.

E-Mail:R.Holzbach@wkp-Iwl.org

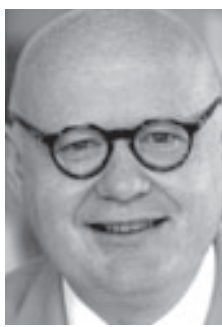

Prof. Dr. med. Frank G. B. Pajonk ist Chefarzt der Privat-NervenKlinik Dr. Kurt Fontheim in Liebenburg. Zu seinen wissenschaftlichen Schwerpunkten zählen Wirkungen von Psychopharmaka und psychiatrische Notfälle. E-Mail: pajonk@klinik-dr-fontheim.de

\section{Literatur online}

Das vollständige Literaturverzeichnis zu diesem Beitrag finden Sie im Internet:

Abonnenten und Nichtabonnenten können unter „www.thieme-connect.de/ejournals“ die Seite der AINS aufrufen und beim jeweiligen Artikel auf „Ergänzendes Material“ klicken hier ist die Literatur für alle frei zugänglich.

Abonnenten können alternativ über ihren persönlichen Zugang an das Literaturverzeichnis gelangen. Wie das geht, lesen Sie unter: http://www.thieme-connect.de/ejournals/ help\#SoRegistrieren 